\section{Optical imaging in microfluidic bioreactors enables oxygen monitoring for continuous cell culture}

\author{
Dhruv Sud, ${ }^{\text {a Geeta Mehta, }{ }^{\text {a }} \text { Khamir Mehta, }}{ }^{\text {b }}$ \\ Jennifer Linderman, ${ }^{\mathrm{a}, \mathrm{b}}$ Shuichi Takayama, ${ }^{\mathrm{a}, \mathrm{c}}$ and \\ Mary-Ann Mycek ${ }^{a, d, e, *}$ \\ aUniversity of Michigan, Dept. of Biomedical Engineering, \\ Ann Arbor, Michigan 48109-2099 \\ ${ }^{b}$ University of Michigan, Dept. of Chemical Engineering, \\ Ann Arbor, Michigan 48109-2099 \\ 'University of Michigan, Macromolecular Science and \\ Engineering Program, Ann Arbor, Michigan 48109-2099 \\ 'University of Michigan, Applied Physics Program, \\ Ann Arbor, Michigan 48109-2099 \\ 'University of Michigan, Comprehensive Cancer Center, \\ Ann Arbor, Michigan 48109-2099
}

Abstract. For the first time, a fluorescence lifetime calibration method for an oxygen-sensitive dye ruthenium $\operatorname{tris}(2,2$ '-dipyridyl) dichloride hexahydrate (RTDP) is applied to image oxygen levels in poly(dimethyl siloxane) (PDMS) bioreactors containing living C2C12 mouse myoblasts. PDMS microsystems are broadly used in bioengineering applications due to their biocompatibility and ease of handling. For these systems, oxygen concentrations are of significance and are likely to play an important role in cell behavior and gene expression. Fluorescence lifetime imaging microscopy (FLIM) bases image contrast on fluorophore excited state lifetimes, which reflect local biochemistry. Unique attributes of the widefield, timedomain FLIM system include tunable excitation (337.1 to $960 \mathrm{~nm}$ ), large temporal dynamic range ( $\geq 600 \mathrm{ps}$ ), high spatial resolution $(1.4 \mu \mathrm{m})$, calibrated detection (0 to $300 \pm 8 \mu \mathrm{M}$ of oxygen), and rapid data acquisition and processing times $(10 \mathrm{~s})$. Oxygen levels decrease with increasing cell densities and are consistent with model outcomes obtained by simulating bioreactor oxygen diffusion and cell proliferation. In single bioreactor loops, FLIM detects spatial heterogeneity in oxygen levels with variations as high as 20\%. The fluorescence lifetime-based imaging approach we describe avoids intensity-based artifacts (including photobleaching and concentration variations) and provides a technique with high spatial discrimination for oxygen monitoring in continuous cell culture systems.

(C) 2006 Society of Photo-Optical Instrumentation Engineers. [DOI: $10.1117 / 1.2355665]$

Keywords: fluorescence microscopy; lifetime sensing; microfluidics; oxygen; molecular imaging; cell culture.

Paper 06105LR received Apr. 23, 2006; revised manuscript received Jul. 27, 2006; accepted for publication Jul. 28, 2006; published online Sep. 28, 2006.

Microfluidic devices have promising applications in cellbased assays and microscale tissue engineering, where spatiotemporal conditions are readily manipulated. Recently,

*Tel: 734647 1361; E-mail: mycek@umich.edu poly(dimethyl siloxane)- (PDMS)-based microfluidic systems have been developed as biocompatible and rapidly prototyped systems for microscale-cell culture. For example, cells could be seeded and cultured successfully under continually perfused conditions to achieve an extracellular fluid-to-cell (volume) ratio close to the physiological value ${ }^{1}$ of 0.5 . This small ratio facilitates heterogeneous chemical distribution, which may be critical in specifying cell fate in developing tissues. It is hence of great interest to quantitatively and with minimal perturbation characterize components (e.g., mitogens, nutrients, oxygen) in microfluidic bioreactors that influence cellular responses.

Oxygen in cell cultures influences cell signaling, growth, differentiation, and death. ${ }^{1}$ PDMS bioreactors are popular due to their high diffusivity of oxygen, which has been repeatedly demonstrated. ${ }^{2}$ It has been observed, ${ }^{3}$ however, that the diffusivity of PDMS can vary, depending on protein adsorption (e.g., when cells are cultured) or surface modification (e.g., plasma oxidization for bioreactors). It is hypothesized that this variability in PDMS permeability, along with cellular uptake and culture media perfusion, can affect spatial variations in oxygen within PDMS bioreactors.

Optical measurements of oxygen sensitive agents have advantages over more traditional, electrode-based approaches that make them uniquely applicable for bioreactor systems: they are well suited for small volumes, are relatively nonperturbing, and do not consume oxygen during the measurement. For long-term cell culture, optical sensing enables time-lapse studies (hours or days) without disturbing the setup, as well as imaging spatial oxygen distributions, which is useful for longterm cell culture.

Fluorescence intensity studies based on the oxygen sensitivity of ruthenium complexes embedded in matrix have been performed; ${ }^{4,5}$ these studies were intensity based, did not employ imaging, and did not report local oxygen concentration variations. Intensity-based fluorescence measurements are sensitive to instrumental variations (changes in excitation intensity or optical loss) and are affected by fluorophore concentration and photobleaching. Fluorescence lifetime, however, is an intrinsic property of the fluorophore's excited electronic state and is insensitive to intensity artifacts. FLIM in microfluidic structures was reported for studying solvent mixing by monitoring viscosity in channels without cells. ${ }^{6}$ In this work, calibration of the oxygen sensitivity of RTDP lifetime $^{7}$ on a unique widefield, time-domain fluorescence lifetime imaging microscopy (FLIM) system $^{8,9}$ was applied for quantitative oxygen estimation. To our knowledge, this is the first demonstration of using a lifetime imaging modality for extracellular oxygen monitoring in PDMS bioreactors containing living cells.

The FLIM system (Fig. 1) employs a tunable, pulsed (800-ps FWHM) excitation source operating in single-shot mode that covered the UV-vis-NIR spectrum (337 to $960 \mathrm{~nm}$ ) and an intensified, gated ( $\geq 200$ ps gate width) CCD camera (Picostar HR, LaVision) to record images, with an intensity reproducibility ${ }^{10}$ within $\pm 2 \%$. The system's optical sectioning capability (via structured illumination) was not required for this study. Lifetime maps had a lateral resolution of $1.4 \mu \mathrm{m}$, which was well suited for studying small microfluidic struc-

1083-3668/2006/11(5)/050504/3/\$22.00 @ 2006 SPIE 


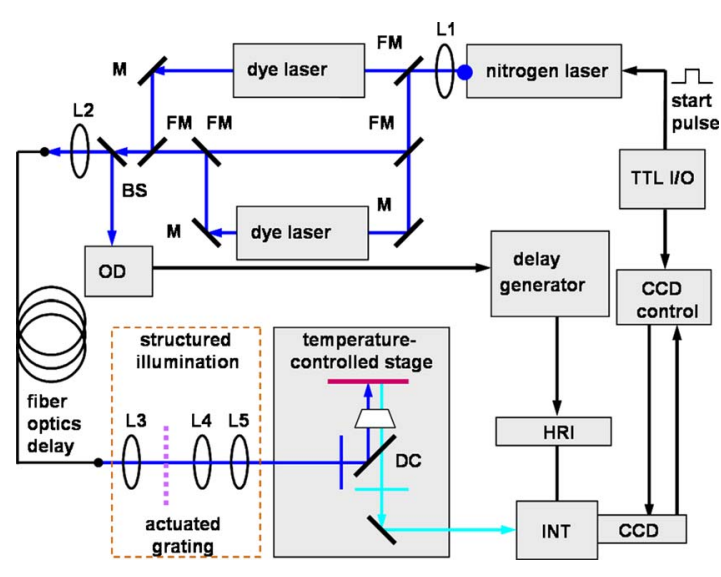

Fig. 1 (Color online only) Widefield, time-domain FLIM instrumentation: CCD, charge-coupled device; HRI, high-rate imager; INT, intensifier; TTL I/O, TTL (transistor-transistor logic) input/output card; OD, optical discriminator; BS, beamsplitter; DC, dichroic mirror; FM, mirror on retractable "flip" mount; L1, L2, L3, L4, and L5, quartz lenses; $M$, mirror; colored thick lines are the optical path and black thick lines are the electronic path.

tures. Lifetimes could be measured in the range of $750 \mathrm{ps}$ to $\infty$, with a temporal discrimination of $50 \mathrm{ps}$.

RTDP [ruthenium tris $\left(2,2^{\prime}\right.$-dipyridyl) dichloride hexahydrate] calibration ${ }^{10}$ at $22^{\circ} \mathrm{C}$ was verified using FLIM and an oxygen sensor ${ }^{7}$ (FOXY, Ocean Optics). Briefly, gas tubing was attached to one port on a perfused lid over a culture dish with RTDP stock solution, while the FOXY probe was inserted into the solution via the other port. Under equilibrium conditions, the oxygen level was recorded as $8.5 \mathrm{mg} / \mathrm{L}$. Nitrogen was flowed over the sample and a drop in oxygen was recorded via FOXY. Once the FOXY output stabilized at $<0.1 \mathrm{mg} / \mathrm{L}$, the lifetime was recorded as $\tau_{0}$. RTDP fluorescence quenching by oxygen is a collisional process described by the Stern-Volmer equation: $\tau_{0} / \tau_{x}=1+K_{q}\left[\mathrm{O}_{2}\right]_{x}$, where $\tau_{0}$ $=$ uninhibited RTDP lifetime (i.e. $0 \%$ oxygen), $\tau_{x}=$ RTDP lifetime at oxygen level $\left[\mathrm{O}_{2}\right]_{x}$, and $K_{q}$ is the Stern-Volmer quenching constant.

For our measurements, $K_{q}$ was determined ${ }^{7}$ to be equal to $2.7 \times 10^{-3} \mu \mathrm{M}^{-1}$. RTDP fluorescence images from bioreactor loops were collected by a $10 \times, 0.3$-numerical aperture (NA) objective (Zeiss, Jena, Germany) at $600 \mathrm{~nm}$ (HQ500lp, Chroma Technology Corp.) emission via 460-nm excitation at gates of $40,140,240,340$, and $440 \mathrm{~ns}$, with the intensifier gate width set to $100 \mathrm{~ns}$. A lifetime macro applying the rapid lifetime determination (RLD) algorithm ${ }^{10}$ was used to generate lifetime images on a per pixel basis from gated intensity images (Fig. 2). Oxygen levels were ascertained by fitting the Stern-Volmer equation to calculated lifetimes at each pixel to generate oxygen distribution images (Fig. 2).

Each PDMS bioreactor was fabricated using backlight soft photolithography. ${ }^{1}$ The seed channel was injected with fibronectin to promote cell attachment. C2C12 cells (mouse myoblasts) were cultured under standard conditions and injected into the channels after being suspended in phosphatebuffered saline $^{1}$ (PBS). RTDP $(3 \mathrm{mg} / \mathrm{ml})$ dissolved in PBS was injected into the media reservoir 1 to $2 \mathrm{~h}$ after cell seeding and the bioreactor was perfused by peristaltic pumping action of an array of pin actuators adapted from Braille dis-

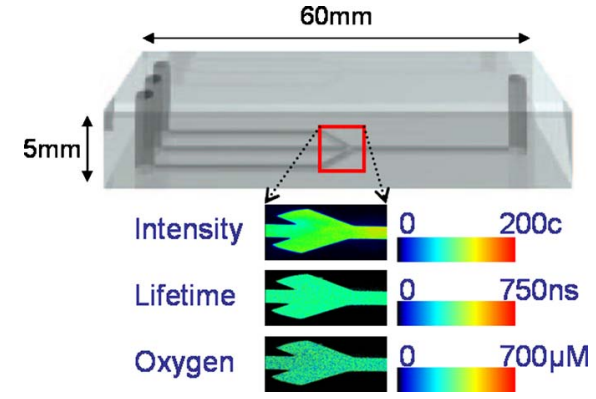

Fig. 2 (Color online only) Illustration of fluorescence intensity and lifetime imaging in microfluidic devices. Top: perspective view of a device that contained $\mathrm{C} 2 \mathrm{C} 12$ mouse myoblasts and was perfused with media containing RTDP at a rate of approximately $0.5 \mathrm{nl} / \mathrm{s}$ by gravitydriven flows. Channel height $=50 \mu \mathrm{M}$, width $=300 \mu \mathrm{m}$. Bottom: representative images of RTDP fluorescence intensity (scale in counts), lifetime (microseconds), and oxygen $(\mu \mathrm{M})$ obtained via FLIM.

plays for three more hours to enable RTDP to distribute along bioreactor channels. ${ }^{1}$

For imaging, the bioreactor was removed from the pumping system and placed on the microscope stage. RTDP yielded a bright signal within the PDMS bioreactor, which by itself was optically transparent. RTDP was well tolerated by the myoblasts within the bioreactor, as evidenced by cytotoxic and phototoxic studies done using trypan blue (SigmaAldrich). A published partial differential equation (PDE) model describing oxygen diffusion, convection, and uptake by cells within the device was developed and solved using ${ }^{11}$ FEMLAB (Consol AB Inc.).

The cell-seeding procedure resulted in variable cellular densities across different microfluidic devices, so a basic bioreactor design was used to study effects of cell density on oxygen levels. Figure 2 illustrates the 3-D design as well as typical intensity, lifetime, and oxygen images that were obtained via FLIM. The results from FLIM studies and computational simulations (accounting for channel geometry) were in good agreement and are shown in Fig. 3. As expected, the observed extracellular oxygen level in the bioreactor decreased in a cell-density-dependent manner by almost 35\% after incubation for $2 \mathrm{~h}$. Oxygen levels measured here are lower compared to conventional macroscopic cell culture systems due to higher cell densities and low media levels. Lowered oxygen levels are still conducive to cell growth in vitro, as indicated by proliferation of cells in the bioreactor over a 12 to $14 \mathrm{~h}$ period (data not shown).

A PDMS cell culture device with six loops is illustrated in Fig. 4(a). The device was small scale, optically transparent, and ideal for microscopic analysis. FLIM images of oxygen levels were taken at different points along each loop, as illustrated in Fig. 4(b). FLIM detection revealed that oxygen levels differed by as much as $20 \%$ within a loop, and this trend was consistently observed across multiple devices. Differences in measurements made at different points within the same loop were statistically significant [analysis of variance (ANOVA) and Student's $t$ test, $p<0.001]$. (Comparisons across loops are not logical since each loop can potentially harbor different cell densities, which, as shown earlier, can significantly affect oxygen levels.) 


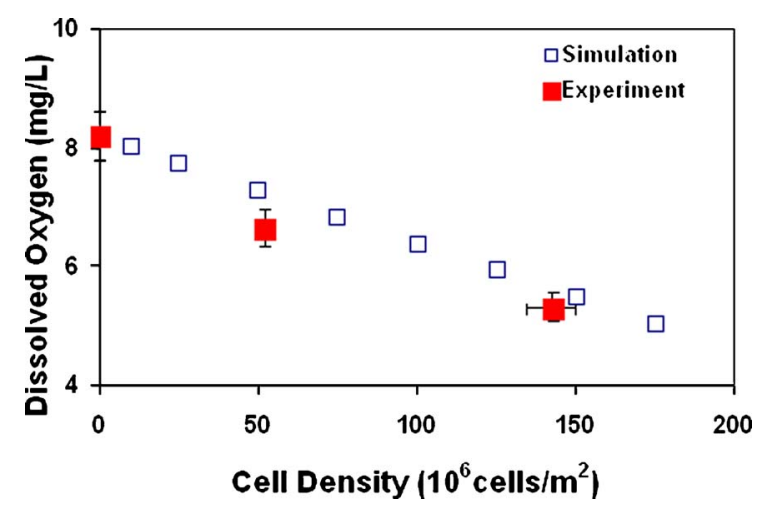

Fig. 3 (Color online only) Simulation (white squares) and experimental FLIM (red squares) results of oxygen levels versus cell densities in channels illustrated in Fig. 2. Oxygen levels were estimated by averaging pixel values in oxygen distribution images of the channel. The model simulations were carried out according to the equations described in Ref. 11, with the model parameters set at maximum oxygen uptake rate $V_{\max }=2 e^{-16} \mathrm{~mol} / \mathrm{cell}^{1} \mathrm{~s}-1$; oxygen level at half-saturation $K_{\mathrm{m}}=0.0059 \mathrm{~mol} / \mathrm{m}^{3}$; overall mass transfer coefficient $k_{l a}$ $=4.5 e^{-7} \mathrm{~m} / \mathrm{s}$, and estimated velocity of gravity flow $\langle u\rangle=0.003 \mathrm{~m} / \mathrm{s}$. Error bars for some experimental data were within the red squares.

In conclusion, FLIM of RTDP was applied for the first time to measure oxygen in microfluidic bioreactors containing living cells. The calibrated method of oxygen estimation was sensitive over the entire physiological range ( 0 to $300 \mu \mathrm{M}$ of oxygen) with a resolution ${ }^{7}$ of $\pm 8 \mu \mathrm{M}$. Measured extracellular oxygen levels correlated with cell densities in the channels and were verified with model simulations (Figs. 2 and 3). Initial results on a bioreactor with six loops (Fig. 4) revealed statistically significant variations in oxygen levels within each bioreactor loop. An implicit assumption in FLIM measure-
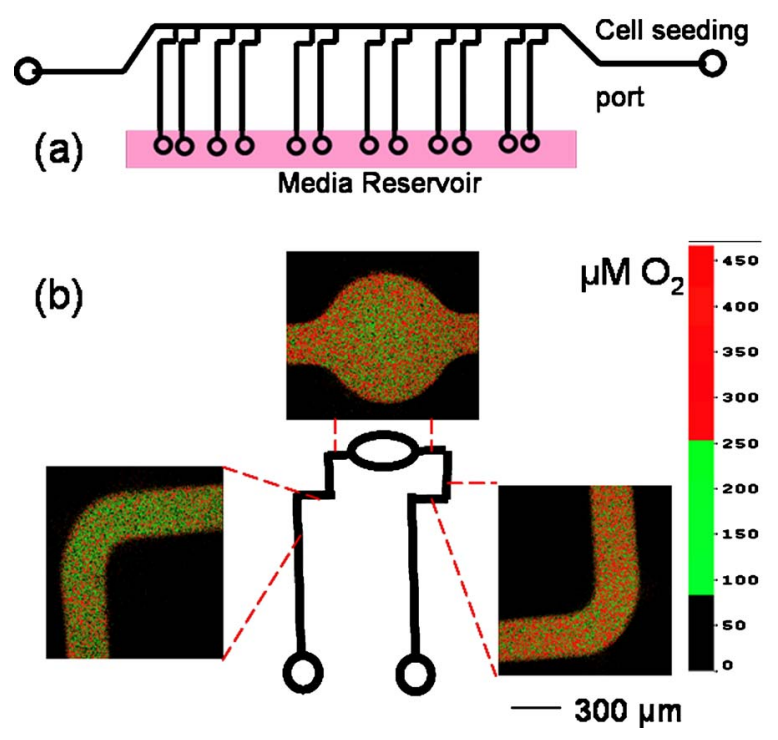

Fig. 4 (Color online only) oxygen measurements from a closed-loop PDMS bioreactor for continuous cell culture of C2C12 mouse myoblasts. (a) Device schematic. Channel shape was an isosceles trapezoid with a height of $30 \mu \mathrm{m}$ and an upper (lower) PDMS layer of $180 \mu \mathrm{m}(402 \mu \mathrm{m})$. Each of the six loops has a right and left valve separating it from the others. (b) Oxygen distribution images at different points of a single loop (binary scale in $\mu \mathrm{M}$ ). ments was that oxygenation did not vary significantly in the axial dimension due to the small height of the channel cross section $(30 \mu \mathrm{m})$, an aspect that has been explored in more detail in simulation scenarios. ${ }^{11}$ Future work will involve long-term oxygen monitoring under continually perfused conditions. Modeling results indicate that media recycling might be beneficial to retain growth factor(s) secreted by cells; ${ }^{11}$ such a scheme will potentially affect oxygen levels, which can be tracked almost in real time due to fast data acquisition $(<10 \mathrm{~s})$ by the FLIM approach. All these endeavors are ultimately aimed at controlling long-term cellular responses (such as differentiation) in PDMS microbioreactors. A molecular, fluorescence-lifetime-based approach to oxygen sensing in PDMS bioreactors, such as that described here, offers several advantages including replacing a macroscopic chemical electrode with a nondestructive (and minimally perturbing) optical imager, which could potentially be customized and scaled-down using optoelectronic technologies, making this approach more economical and portable.

\section{Acknowledgments}

This material is based on work supported by the U.S. Army Research Laboratory and the U.S. Army Research Office under Contract/Grant No. DAAD19-03-1-0168, the National Science Foundation (BES-0238625), and the Whitaker Foundation.

\section{References}

1. W. Gu, X. Y. Zhu, N. Futai, B. S. Cho, and S. Takayama, "Computerized microfluidic cell culture using elastomeric channels and Braille displays," Proc. Natl. Acad. Sci. U.S.A. 101(45), 15861-15866 (2004).

2. E. Leclerc, Y. Sakai, and T. Fujii, "Cell culture in 3-dimensional microfluidic structure of PDMS (polydimethylsiloxane)," Biomed. Microdevices 5(2), 109-114 (2003).

3. H. Shiku, T. Saito, C. C. Wu, T. Yasukawa, M. Yokoo, H. Abe, T. Matsue, and H. Yamada, "Oxygen permeability of surface-modified poly(dimethylsiloxane) characterized by scanning electrochemical microscopy," Chem. Lett. 35(2), 234-235 (2006).

4. F. G. Gao, J. M. Fay, G. Mathew, A. S. Jeevarajan, and M. M. Anderson, "Optical sensor based on fluorescent quenching and pulsed blue LED excitation for long-term monitoring of dissolved oxygen in NASA space bioreactors," J. Biomed. Opt. 10, 054005 (2005).

5. A. Sin, K. C. Chin, M. F. Jamil, Y. Kostov, G. Rao, and M. L. Shuler, "The design and fabrication of three-chamber, microscale cell culture analog devices with integrated dissolved oxygen sensors," Biotechnol. Prog. 20(1), 338-345 (2004).

6. R. K. P. Benninger, O. Hofmann, J. McGinty, J. Requejo-Isidro, I Munro, M. A. A. Neil, A. J. deMello, and P. M. W. French, "Timeresolved fluorescence imaging of solvent interactions in microfluidic devices," Opt. Express 13(16), 6275-6285 (2005).

7. D. Sud, W. Zhong, D. G. Beer, and M. A. Mycek, "Time-resolved optical imaging provides a molecular snapshot of altered metabolic function in living human cancer cell models," Opt. Express 14(10), 4412-4426 (2006).

8. P. K. Urayama, W. Zhong, J. A. Beamish, F. K. Minn, R. D. Sloboda, K. H. Dragnev, E. Dmitrovsky, and M.-A. Mycek, "A UV-visible fluorescence lifetime imaging microscope for laser-based biological sensing with picosecond resolution," Appl. Phys. B 76(5), 483-496 (2003).

9. W. Zhong, P. Urayama, and M.-A. Mycek, "Imaging fluorescence lifetime modulation of a ruthenium-based dye in living cells: the potential for oxygen sensing," J. Phys. D 36(14), 1689-1695 (2003).

10. H. C. Gerritsen, R. Sanders, A. Draaijer, and Y. K. Levine, "Fluorescence lifetime imaging of oxygen in living cells," J. Fluoresc. 7(1), 11-16 (1997).

11. K. Mehta and J. J. Linderman, "Model-based analysis and design of a microchannel reactor for tissue engineering," Biotechnol. Bioeng. 94(3), 596-609 (2006). 